\title{
WeB REVIEWS
}

\section{Web-review: Oncology}

\author{
IK Indrajit \\ Department of Radiodiagnosis \& Imaging, Command Hospital (Air Force), Bangalore - 560007, India \\ Correspondence: Dr. I K Indrajit, Department of Radiodiagnosis and Imaging, Command Hospital (Air Force), Bangalore - 560007, India. \\ E-mail: inji63@gmail.com
}

Few useful websites dealing with oncology and related topics that are available at the moment on the Internet are reviewed below.

1. International Union Against Cancer (UICC) is 'the leading international nongovernmental organization dedicated to the global prevention and control of cancer,' and is headquartered at Geneva. The web portal available at http://www.uicc.org/ has different sections on books, reports, newsletters, position papers, and statements and a link to 'International Journal of Cancer.' The Annual Report 2009 is on offer at http://www.uicc. org/resources/annual-report-2009. An interesting section on 'How to use the TNM classification' is accessible from http://www.uicc.org/resources/how-use-tnmclassification.

2. Cancer Network available at http://www.cancernetwork. com is a 'feature rich' source of information on cancer. The site has useful sections on handbooks, conference reports, podcasts, continuing medical education courses on a variety of cancers, and links to journals like Oncology and Oncology News International.

3. Cancer Screening Programmes available at http://www. cancerscreening.nhs.uk is a portal focusing on Cancer Screening by NHS, United Kingdom. The current programmes focus on breast, cervical, colorectal, and prostate cancer. Useful downloadable documents are available too, assisting the important concept of screening.

4. Cancer.Gov available at http://www.cancer.gov/ is a cancer-related health information portal from the National Cancer Institute. The PDQ (Physician Data Query), NCI's Comprehensive Cancer Database offering the latest information about cancer treatment, screening, prevention, is available at http://www.cancer.gov/ cancertopics/pdq. Likewise, Cancer Literature Search available at http://www.cancer.gov/search/cancer_ literature/ is a comprehensive bibliographic repository resourced from 'biomedical journals, proceedings, books, reports, and doctoral theses.'
5. National Comprehensive Cancer Network is available at http://www.nccn.org/index.asp; the website of the organization NCCN endeavors to 'improve the quality of life for patients and reduce cancer-related deaths by advancing cancer therapies through research.' Registration and access to the NCCN Guidelines ${ }^{\mathrm{TM}}$ and Clinical Resources is free.

6. Cancer.org is the website of American Cancer Society available at http://www.cancer.org/. The society through their website provide useful material targeting medical professionals, including information on cancer type, early detection, prevention, treatment, staging, clinical trials, medical references, and resources. Cancer facts and figures are available at http://www.cancer.org/ Research/CancerFactsFigures/index.

7. Cancer Net available at http://www.cancer.net/portal/ site/patient is an offer from the American Society of Clinical Oncology. Sections on cancer types, publications and resources, news and meetings are available.

8. OncoLink is a portal focusing on Cancer from University of Pennsylvania. OncoLink has several educative sections like types of cancer, updates on cancer treatment, and news on advances in research. From the sitemap at http:// www.oncolink.org/about/sitemap.cfm, a whole range of educative resources may be accessed.

9. Indian Cancer Society available at http://www. indiancancersociety.org/ is the web portal of the organization Indian Cancer Society. The Society headquartered at Lady Ratan Tata Medical and Research Centre, Bombay, publishes "The Indian Journal of Cancer" which is the a professional cancer journal published in India.

10. RECIST (Response Evaluation Criteria in Solid Tumors) are criteria that define the objective response in solid cancers during treatment as well as in validating clinical trials. Emerging recently, they are an outcome of an international collaboration between expert agencies like the European Organization for Research and Treatment of Cancer (EORTC), National Cancer 
Institute (NCI), and National Cancer Institute of Canada Clinical Trials Group. Its website is available at http:// www.recist.com/index.html. A comparison between the current version RECIST 1.1 (January 2009) and the earlier RECIST version 1.0 is available at http://www. recist.com/recist-comparative/index.html.

\section{Endpiece}

Cancer Staging available at http://www.cancerstaging. info/ is an interactive cancer staging program, for which registration is free. The cancer staging tool of the website facilitates staging cancers accurately and provides information on different cancer types. Tumor Atlas available at http://www.bioscience.org/atlases/tumpath/index.htm is a copyrighted contribution obtained from the 'Frontiers in Bioscience,' and is authored by Tabibzadeh et al. The material is an illustrative work dealing with a variety of tumors. Each tumor is further discussed under classification, risk factors, clinical features, images, diagnosis, and TNM Staging, interactively.

eMedicine is a multi-authored, multi-reviewed clinical knowledge base. Launched in 1996, the site presently has numerous authors/editors contributing articles on a large number of diseases and disorders. The specialty of Oncology is covered specifically at http://emedicine. medscape.com/oncology. Furthermore, Medscape, another medical knowledge database, has a specialty home page on Cancer and Oncology, which is available at htp://www. medscape.com/oncology.

Advance for Imaging and Radiation Oncology is a periodical available at http://imaging-radiation-oncology. advanceweb.com/, which provides comprehensive, upto-date news and information on management strategies, clinical/technological updates, and industry trends in medical imaging and radiation oncology. 